\title{
Género y barreras de accesibilidad al tratamiento en pacientes adictos al alcohol de la Comunidad Valenciana
}

\author{
Sofía Tomás-Dols*; Juan Carlos Valderrama-Zurián**; Antonio Vidal-Infer***; Teresa Samper-Gras***; \\ Ma Carmen Hernández-Martínez***; Ma José Torrijo-Rodrigo***. \\ *Dirección General de Drogodependencias. Agencia Valenciana de Salud. Conselleria de Sanitat. Generalitat Valenciana. \\ **Instituto de Historia de la Ciencia y Documentación López Piñero. Universitat de València-CSIC. \\ ** Fundación para el Estudio, Prevención y Asistencia a las Drogodependencias. \\ Enviar correspondencia a: \\ Ma José Torrijo Rodrigo. FEPAD. C/ Guardia Civil 30, entlo. B. 46020 Valencia. E-Mail: pepa.torrijo@fepad.es
}

Recibido: Noviembre de 2006.

Aceptado: Febrero de 2007.

\section{RESUMEN}

Introducción: el objetivo principal del presente estudio fue la identificación de los motivos que retrasan el acceso de los pacientes dependientes de alcohol de la Comunidad Valenciana a centros específicos de tratamiento. Método: fueron entrevistados 563 pacientes de Unidades de Conductas Adictivas y Unidades de Alcohología. Se les administró una encuesta sobre tratamientos previos en otros centros y sobre barreras que retrasan el acceso al tratamiento en centros ambulatorios específicos. Se realizó un análisis descriptivo de los ítems y se utilizó la t de student y el test de ANOVA con prueba de Scheffé. Resultados: el 59,7\% de los entrevistados manifestaba haber solicitado previamente tratamiento en algún centro no especializado en drogodependencias por alguna molestia física o psíquica que actualmente considera relacionada con el consumo de alcohol o drogas y que en su momento no atribuyó al mismo, o por el propio consumo de sustancias (42,8\%). Los servicios más consultados fueron Atención Primaria y Especializada, presentando las mujeres una mayor demanda de tratamiento en los Centros de Salud Mental_( $p<0,05)$. La barreras de tratamiento más importantes estaban incluidas en el eje "no conciencia de enfermedad ni problemas asociados" que obtuvo la mayor puntuación media $(2,2 ; d t=0,6)$. Las mujeres presentaban puntuaciones medias más elevadas en los ejes "estigmatización y respuesta del entorno" y "factores intrínsecos al tratamiento". Conclusiones: existen diferencias intergénero en las barreras que retrasan el acceso a tratamiento. Es necesario elaborar protocolos de intervención adaptados al género de los pacientes para utilizar en centros de Atención Primaria y de Salud Mental y que permitan disminuir las barreras de accesibilidad al tratamiento.

Palabras clave: barreras del tratamiento, dependencia alcohol, accesibilidad, centros de tratamiento en drogodependencias, género.

\section{ABSTRACT}

Introduction: this study aimed at identifying the factors that contribute to delaying the access of alcohol abuse patients to specific treatment centres in the Autonomous Region of Valencia (Spain). Method: 563 patients from Addictive Behaviours Units (UCA) and Alcohology Units (UA) were interviewed. A survey was conducted which included items on previous requested treatment in other centres and on barriers of accessibility to treatment in specific ambulatory centres. A descriptive analysis and t-student and ANOVA with Scheffé post-hoc tests were carried out. Results: $59.7 \%$ of respondents said they had requested previous treatment in non-specific resources due to physical or psychical trouble that they now relate to their alcohol use although they did not do so at the time, in addition to being motivated by their own alcohol abuse (42.8\%). The most attended resources were Primary Care and Specialist Unit Care. Women showed a higher demand for treatment in Mental Health Services $(p<0,05)$. The most important treatment barriers were included in the axis "unawareness of illness and related problems" $(2.2 ; \mathrm{dt}=0,6)$. Women obtained higher scores in the axes "stigmatisation and environment response" and "treatment intrinsic factors". Conclusions: gender differences in barriers that delay access to treatment do exist. It is necessary to build genderadapted intervention guidelines to be used in Primary Care and Mental Health services to reduce the accessibility barriers to treatment.

Key words: treatment barriers, alcohol dependence, accessibility, drug abuse treatment centres, gender. 


\section{INTRODUCCIÓN}

E consumo excesivo de alcohol es en la actuaidad un importante problema de salud pública y genera un elevado número de enfermedades orgánicas y psicosociales ${ }^{1}$. Según la Organización Mundial de la Salud, los problemas relacionados con el consumo de alcohol contribuyeron en un $4 \%$ a la morbilidad mundial en el año 2002². En la Comunidad Valenciana, el consumo de alcohol se mantiene en los últimos años en cifras estables, siendo la prevalencia en población general de 15 a 65 años de consumo en los últimos 30 días de 6,1\% en 1998 y de 6,3\% en $2004^{3,4}$

En la Comunidad Valenciana existen recursos específicos para la asistencia ambulatoria al paciente alcohólico como son las Unidades de Conductas Adictivas (UCA) y las Unidades de Alcohología (UA) creadas por la Conselleria de Sanidad y que están incluidas en el sistema sanitario público ${ }^{5}$. Entre las funciones de estas Unidades están el diagnóstico, el tratamiento farmacológico y psicoterapéutico de los pacientes con patología adictiva y la coordinación con los distintos recursos sanitarios y sociales. La evolución de las demandas de tratamiento por consumo de alcohol en estos centros ha experimentado un incremento del $36 \%$ en los últimos años pasando de 2.928 demandas en 1998, a 3.981 en 20046, siendo, aproximadamente el $20 \%$ de las demandas realizadas por mujeres con dependencia de alcohol.

El tratamiento ambulatorio del alcoholismo permite controlar de una manera segura la sintomatología de abstinencia mediante desintoxicaciones ambulatorias ${ }^{7}$ y produce una mejoría de la salud física y mental, de las relaciones familiares y sociales y del funcionamiento general del paciente ${ }^{8,9}$, es decir, una mejora significativa del mismo ${ }^{10}$. Así, el tratamiento del alcoholismo disminuye la asistencia a los servicios de urgencias y el número de días de ingreso hospitalario ${ }^{11}$, siendo necesario adaptar los tratamientos al género de los pacientes ${ }^{12-14}$. No obstante, estudios previos demuestran que tan sólo una pequeña parte de los sujetos con problemas relacionados con el consumo de alcohol utiliza los recursos sociosanitarios disponibles ${ }^{15-19}$ y que los pacientes con dependencia de sustancias suelen tardar más en acudir a los centros asistenciales que las personas con trastornos mentales ${ }^{20}$, situándose en al menos 10 años el tiempo desde que se instaura la dependencia hasta que se demanda tratamiento ${ }^{21}$.

La accesibilidad al tratamiento es uno de los pilares básicos de una buena asistencia sanitaria y está considerada como uno de los elementos más importantes en la calidad asistencial ${ }^{2-24}$. Sin embargo, existen factores que pueden impedir o retrasar el acceso a un programa de tratamiento del alcoholismo como son los de tipo personal25, los problemas físicos y psíquicos relacionados con el consumo de alcohol ${ }^{26}$, los relacionados con la personalidad del paciente ${ }^{27}$ y la no adecuación de los programas de tratamiento a las características de los pacientes, tales como la edad ${ }^{28}$, la etnia ${ }^{17}$, la disponibilidad económica ${ }^{29}$ o la distancia geográfica al centro de tratamiento ${ }^{30}$. También hay factores intrínsecos del servicio sanitario que pueden retrasar el acceso al tratamiento como es la falta de tiempo del personal ${ }^{31}$. Asimismo, se han encontrado en otros países diferencias en las barreras al tratamiento o en el retraso en la asistencia a los Centros específicos de drogodependencias según el género de los pacientes ${ }^{32}, 33$.

La identificación de dichos factores puede facilitar el acceso de los potenciales usuarios a los recursos específicos de tratamiento. Por ello, el objetivo principal del presente trabajo es estudiar los principales motivos que pueden retrasar la solicitud de tratamiento de pacientes adictos al alcohol en los centros de drogodependencias de atención ambulatoria de la Comunidad Valenciana y analizar si existen diferencias según el género.

\section{MÉTODO}

\section{Diseño del estudio}

La población diana estaba compuesta por pacientes de 18 a 65 años que habían iniciado tratamiento por dependencia de alcohol en UCA o UA de la Comunidad Valenciana o llevaban como máximo doce meses en tratamiento en dichas unidades por consumo de alcohol. El estudio se llevó a cabo en 25 UCA y UA de la Comunidad Valenciana, lo que representa un $73,52 \%$ del total de unidades. Estos centros disponen al menos de un profesional médico y un psicólogo. El trabajo de campo se desarrolló durante el mes de noviembre de 2004. Todos los participantes del estudio fueron entrevistados de manera presencial en los centros y la entrevista duraba aproximadamente 15 minutos. A todos los sujetos se les requirió el consentimiento informado para que formaran parte del estudio y se les garantizó el anonimato y la confidencialidad.

\section{Muestra}

La muestra se obtuvo mediante un muestreo aleatorio sistemático, estratificándose la muestra en cada UCA en base a la demanda asistencial durante los primeros nueve meses de 2004. El tamaño muestral se estableció en 526 sujetos, para un error genérico de muestreo de $\pm 4 \%$ con un intervalo de confianza del $95 \%$. 
Finalmente participaron en el estudio 563 individuos, $76,2 \%$ hombres y $23,8 \%$ mujeres, con una edad media de 43,2 años $(d t=9,2)$, siendo la edad media de los hombres de 43 años $(\mathrm{dt}=9,0)$ y de las mujeres de 43,8 años ( $d t=9,7)$.

\section{Instrumento}

El instrumento de recogida de información se estructuró en dos grandes apartados: el primero recogía datos básicos (sexo, edad, tiempo de tratamiento - estableciéndose para el análisis posterior los siguientes rangos: menos de 1 mes, de 1 a 3 meses, 4 a 6 meses, 7 a 9 meses y 10 a 12 meses- y existencia de contacto previo con los servicios sanitarios motivado por el propio consumo de drogas o por algún motivo relacionado con dicho consumo) y otro apartado compuesto por 50 ítems de tipo Likert, puntuados según el grado de influencia de los factores como barreras para el tratamiento y evaluados en una escala de $1=$ nada a 4 =mucho. El listado de motivos se elaboró basándose en estudios previos: la National Survey on Drug Use and Health del año $2002^{34}$ y las distintas versiones del The Barriers Questionnaire desarrollado por Miller y Tonigan ${ }^{35}$. Para el análisis posterior de los datos se agruparon los ítems en cinco ejes en base al contenido implícito de las preguntas: no conciencia de enfermedad y problemas asociados, resistencia al cambio, factores intrínsecos al tratamiento, factores extrínsecos al tratamiento y estigmatización y respuesta del entorno.

\section{Análisis estadístico}

Los datos fueron analizados mediante el programa estadístico SPSS versión 12.0. Se realizo un análisis descriptivo y de frecuencias de las variables. Para la comparación de frecuencias se utilizó la chi-cuadrado y para la comparación de medias la t de Student. Asimismo, se analizó si se obtenían diferencias estadísticamente significativas en las puntuaciones de los diversos ítems según el tiempo en tratamiento o los recursos sanitarios utilizados por el consumo de alcohol. Para ello se utilizó un test ANOVA, seguido de la prueba de contraste post-hoc de Scheffé. El nivel de significación estadística se estableció en $p<0,05$.

\section{RESULTADOS}

\section{Solicitudes previas de tratamiento}

El 59,7\% de los entrevistados manifestaba haber solicitado previamente tratamiento en algún centro no especializado en drogodependencias por alguna molestia física o psíquica que actualmente considera relacionada con el consumo de alcohol o drogas y que en su momento no atribuyó al mismo $(49,4 \%)$ o por el propio consumo de sustancias (42,8\%). Los servicios más consultados fueron Atención Primaria y Especializada (tabla 1). Las mujeres presentaban una mayor demanda de tratamiento que los hombres en los Centros de Salud Mental_por alguna molestia física o psíquica que actualmente considera relacionada con el consumo de alcohol o drogas y que en su momento no atribuyó al mismo $(p<0,001)$ o por el consumo de alcohol o sustancias $(p<0,05)$.

Tabla 1: Características de la muestra según edad, tiempo en tratamiento y demandas previas de tratamiento.

\begin{tabular}{|c|c|c|c|c|}
\hline & & Hombres & Mujeres & Total \\
\hline \multicolumn{2}{|l|}{$\mathbf{N}(\%)$} & $429(76,2 \%)$ & $134(23,8 \%)$ & $563(100,0 \%)$ \\
\hline \multicolumn{2}{|l|}{ Edad media (dt) } & $43,0(9,0)$ & $43,8(9,7)$ & $43,2(9,2)$ \\
\hline \multicolumn{2}{|c|}{ Periodo medio de tratamiento (meses) } & 5,2 & 4,9 & 5,1 \\
\hline \multicolumn{2}{|c|}{$\begin{array}{l}\text { Porcentaje de pacientes que solicitaron tratamiento antes } \\
\text { en otro tipo de servicio sanitario por alguna molestia física o } \\
\text { psíquica que ahora considera que estaba relacionada con su } \\
\text { consumo de alcohol u otras drogas y que en su momento no } \\
\text { atribuyó al mismo }\end{array}$} & 48,7 & 51,5 & 49,4 \\
\hline \multirow{5}{*}{$\begin{array}{l}\text { Centros donde } \\
\text { lo solicitaron }\end{array}$} & Atención Primaria & 27,0 & 26,9 & 27,0 \\
\hline & Especialista & 16,1 & 9,0 & 14,4 \\
\hline & Salud Mental & 10,0 * & 22,4 & 13,0 \\
\hline & Urgencias & 7,2 & 6,7 & 7,1 \\
\hline & Otros & 9,8 & 5,2 & 8,7 \\
\hline
\end{tabular}




\begin{tabular}{|c|c|c|c|c|}
\hline \multicolumn{2}{|c|}{$\begin{array}{l}\text { Porcentaje de pacientes que solicitaron tratamiento antes en } \\
\text { otro tipo de servicio sanitario por su consumo de alcohol u } \\
\text { otras drogas. }\end{array}$} & 42,4 & 44,0 & 42,8 \\
\hline \multirow{5}{*}{$\begin{array}{l}\text { Centros donde } \\
\text { lo solicitaron }\end{array}$} & Atención Primaria & 21,2 & 26,9 & 22,6 \\
\hline & Especialista & 10,3 & 9,7 & 10,1 \\
\hline & Salud Mental & $9,3 * *$ & 17,2 & 11,2 \\
\hline & Urgencias & 6,3 & 4,5 & 5,9 \\
\hline & Otros & 15,2 & 14,2 & 14,9 \\
\hline \multicolumn{2}{|c|}{$\begin{array}{l}\text { Porcentaje de pacientes que solicitaron tratamiento con } \\
\text { anterioridad en otro servicio sanitario. }\end{array}$} & 58,0 & 64,9 & 59,7 \\
\hline \multirow{5}{*}{$\begin{array}{l}\text { Centros donde } \\
\text { lo solicitaron }\end{array}$} & Atención Primaria & 35,0 & 39,6 & 36,1 \\
\hline & Especialista & 19,6 & 15,7 & 18,7 \\
\hline & Salud Mental & $13,3^{* * *}$ & 30,6 & 17,4 \\
\hline & Urgencias & 10,7 & 10,4 & 10,7 \\
\hline & Otros & 17,0 & 16,4 & 16,9 \\
\hline
\end{tabular}

${ }^{*}\left(X^{2}=13,8, g \mid=1, p<0,001\right)$

$* *\left(X^{2}=6,3, g l=1, p<0,05\right)$

$* * *\left(X^{2}=21,28, g l=1, p<0,001\right)$

Los pacientes que no habían solicitado previamente tratamiento presentaban puntuaciones más bajas en los ejes de "no conciencia de enfermedad y problemas asociados", "resistencia al cambio", "factores intrínsecos al tratamiento" y "estigmatización y respuesta del entorno". Se obtuvieron diferencias estadísticamente significativas respecto al grupo de sujetos que había acudido previamente a algún servicio por molestia que ahora relacionan con el consumo y en su momento no atribuyeron al mismo o que habían acudido por el propio consumo (tablas 2 y 3 ).

Tabla 2: Análisis de varianza de los ejes según demandas previas de tratamiento

\begin{tabular}{|c|c|c|c|c|c|}
\hline & $\begin{array}{l}\text { Suma de } \\
\text { cuadrados }\end{array}$ & gl & $\begin{array}{c}\text { Media } \\
\text { cuadrática }\end{array}$ & $\mathrm{F}$ & Sig. \\
\hline Eje 1: No conciencia de enfermedad ni problemas asociados & 7,3 & 3 & 2,4 & 6,2 & 0,000 \\
\hline Eje 2: Resistencia al cambio & 5,1 & 3 & 1,7 & 5,2 & 0,001 \\
\hline Eje 3: Factores extrínsecos al tratamiento & 0,5 & 3 & 0,2 & 1,2 & 0,289 \\
\hline Eje 4: Factores intrínsecos al tratamiento & 1,9 & 3 & 0,6 & 5,2 & 0,002 \\
\hline Eje 5: Estigmatización y respuesta del entorno & 1,9 & 3 & 0,6 & 4,0 & 0,008 \\
\hline
\end{tabular}


Tabla 3: Diferencias en los ejes según demandas previas de tratamiento (Scheffé)

\begin{tabular}{|c|c|c|c|c|c|}
\hline & & C1 $(n=227)$ & $\mathbf{C 2}(n=95)$ & C3 $(n=58)$ & C4 $(n=183)$ \\
\hline No conciencia de enfermedad y problemas relacionados & $\begin{array}{l}\text { C1 } \\
\text { C2 } \\
\text { C3 } \\
\text { C4 }\end{array}$ & $\begin{array}{l}p=0,001 \\
\quad N S \\
\text { NS }\end{array}$ & $\begin{array}{l}\text { NS } \\
\text { NS }\end{array}$ & NS & \\
\hline 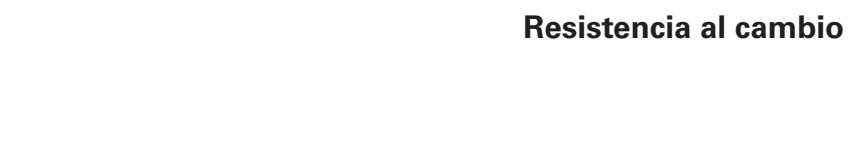 & $\begin{array}{l}\text { C1 } \\
\text { C2 } \\
\text { C3 } \\
\text { C4 }\end{array}$ & $\begin{array}{l}p=0,005 \\
\quad N S \\
\text { NS }\end{array}$ & $\begin{array}{l}\text { NS } \\
\text { NS }\end{array}$ & NS & \\
\hline Factores extrínsecos al tratamiento & $\begin{array}{l}\text { C1 } \\
\text { C2 } \\
\text { C3 } \\
\text { C4 }\end{array}$ & $\begin{array}{l}\text { NS } \\
\text { NS } \\
\text { NS }\end{array}$ & $\begin{array}{l}\text { NS } \\
\text { NS }\end{array}$ & NS & \\
\hline Factores intrínsecos al tratamiento & $\begin{array}{l}\text { C1 } \\
\text { C2 } \\
\text { C3 } \\
\text { C4 }\end{array}$ & $\begin{array}{c}p=0,039 \\
N S \\
p=0,006\end{array}$ & $\begin{array}{l}\text { NS } \\
\text { NS }\end{array}$ & NS & \\
\hline Estigmatización y respuesta del entorno & $\begin{array}{l}\text { C1 } \\
\text { C2 } \\
\text { C3 } \\
\text { C4 }\end{array}$ & $\begin{array}{c}N S \\
N S \\
p=0,010\end{array}$ & $\begin{array}{l}\text { NS } \\
\text { NS }\end{array}$ & NS & \\
\hline
\end{tabular}

NS: No significativo

Se establecieron cuatro grupos en función de las demandas previas de tratamiento, asignando al grupo C1 aquellos individuos que no acudieron previamente a un recurso no especializado por el consumo de alcohol, al grupo C2 quienes acudieron a un recurso no especializado por una molestia que ahora relacionan con el consumo de sustancias y que en su momento no atribuyeron al mismo, al grupo C3 aquellos que acudieron a un recurso no especializado por su consumo de sustancias y, por último, al grupo C4 a quienes acudieron a recursos no especializados, tanto por molestias que ahora relacionan con el consumo de sustancias y que en su momento no atribuyeron al mismo, como por el propio consumo de sustancias.

\section{Barreras al tratamiento}

La puntuación media de los ejes analizados y de cada uno de los ítems se observa en la tabla 4. El eje "no conciencia de enfermedad ni problemas asociados" obtuvo la mayor puntuación media $(2,2 ; \mathrm{dt}=0,6)$, mientras que el eje que presentaba una menor puntuación media era el de "factores extrínsecos al tratamiento" $(1,4 ; d t=0,4)$. Los ítems que presentaron una mayor puntuación fueron: "pensó que podría superarlo personalmente, con sus propios medios" (2,6; $\mathrm{dt}=1,0)$ "creía que los tratamientos eran para personas que estaban peor que usted" $(2,4 ; d t=1,1)$ "no pensó que tuviera un problema serio con las drogas" $(2,3 ; \mathrm{dt}=1,0)$, "no pensaba que necesitara ayuda" $(2,2 ; \mathrm{dt}=1,0)$ y "se encontraba avergonzado/a" $(2,2$; $d t=1,1)$. Por su parte, las barreras que consideraban menos importantes eran la no atención en UCA/UA de una solicitud anterior $(1,0 ; d t=0,3)$, tener el seguro sanitario privado y no creer que pudiera ser atendido/a en la sanidad pública $(1,1 ; d t=0,5)$, malas experiencias en tratamiento de conocidos $(1,1 ; \mathrm{dt}=0,5)$, desaprobación por parte de algún familiar o amigo a comenzar un tratamiento $(1,1 ; d t=0,5)$ y la posibilidad de que iniciar un tratamiento pudiera suponer un problema legal $(1,1 ; d t=0,5)$. No se observaron diferencias estadísticamente significativas en las puntuaciones obtenidas en cada uno de los ítems según el tiempo que actualmente llevaban los pacientes en tratamiento. 
Tabla 4: Diferencia según sexo en las puntuaciones medias obtenidas en cada uno de los ítems y de los ejes.

\begin{tabular}{|c|c|c|c|c|c|}
\hline & Total & $\begin{array}{l}\text { HOM- } \\
\text { BRES }\end{array}$ & MUJERES & $\mathbf{t}$ & Sig \\
\hline EJE I: No conciencia de enfermedad y problemas relacionados & $2,2(0,6)$ & $2,3(0,6)$ & $2,1(0,6)$ & & \\
\hline A su juicio, las drogas no eran su problema principal. & $2,1(1,1)$ & $2,1(1,1)$ & $2,1(1,1)$ & & \\
\hline Su consumo de drogas le parecía normal. & $2,1(1,0)$ & $2,2(1,0)$ & $1,9(0,9)$ & 3,0 & 0,003 \\
\hline $\begin{array}{l}\text { Creía que los tratamientos eran para personas que estaban peor que } \\
\text { usted. }\end{array}$ & $2,4(1,1)$ & $2,5(1,1)$ & $2,2(1,1)$ & 2,4 & 0,015 \\
\hline A su juicio, el consumo de drogas no le generaba ningún problema. & $2,2(1,0)$ & $2,2(1,0)$ & $2,1(1,0)$ & & \\
\hline No creía que fuera una persona drogodependiente. & $2,2(1,1)$ & $2,1(1,1)$ & $2,2(1,1)$ & & \\
\hline Pensó que podía superarlo personalmente, con sus propios medios. & $2,6(1,0)$ & $2,6(1,0)$ & $2,5(1,0)$ & & \\
\hline No pensó que tuviera un problema serio con las drogas. & $2,3(1,0)$ & $2,3(1,0)$ & $2,3(1,1)$ & & \\
\hline No pensaba que necesitara ayuda. & $2,2(1,0)$ & $2,2(1,0)$ & $2,2(1,0)$ & & \\
\hline $\begin{array}{l}\text { Las drogas no le habían causado, realmente, muchos problemas en su } \\
\text { vida. }\end{array}$ & $2,2(1,1)$ & $2,2(1,1)$ & $2,0(1,0)$ & & \\
\hline No pensaba que fuera una enfermedad. & $2,1(1,1)$ & $2,2(1,1)$ & $2,1(1,1)$ & & \\
\hline EJE 2: Resistencia al cambio & $2,0(0,6)$ & $2,0(0,6)$ & $2,0(0,6)$ & & \\
\hline No le gusta que nadie le diga lo que tiene que hacer con su vida. & $2,1(1,0)$ & $2,2(1,0)$ & $2,1(1,0)$ & & \\
\hline $\begin{array}{l}\text { Consumir alcohol u otras drogas le aportaba más cosas buenas que } \\
\text { malas. }\end{array}$ & $1,9(1,0)$ & $1,9(0,9)$ & $2,0(1,0)$ & & \\
\hline Creía que no sabría vivir sin consumir drogas. & $1,9(1,0)$ & $1,8(1,0)$ & $2,1(1,0)$ & $-2,6$ & 0,009 \\
\hline $\begin{array}{l}\text { Le preocupaba las molestias que pudiera causarle el síndrome de abstinen- } \\
\text { cia. }\end{array}$ & $1,9(1,0)$ & $1,9(1,0)$ & $2,0(1,0)$ & & \\
\hline Le gustaba consumir drogas y no quería dejar de hacerlo. & $2,1(1,0)$ & $2,1(1,0)$ & $2,1(1,0)$ & & \\
\hline Creía que el tratamiento no serviría para nada. & $1,6(0,9)$ & $1,6(0,9)$ & $1,6(0,9)$ & & \\
\hline No quería dejar de consumir. & $2,1(1,0)$ & $2,1(1,0)$ & $2,1(1,0)$ & & \\
\hline EJE 3: Factores extrínsecos al tratamiento & $1,4(0,4)$ & $1,4(0,4)$ & $1,4(0,4)$ & & \\
\hline No podía conseguir permiso en el trabajo para ir a tratamiento. & $1,3(0,8)$ & $1,4(0,8)$ & $1,2(0,6)$ & 2,5 & 0,011 \\
\hline Tenía dificultades de transporte para ir a tratamiento. & $1,1(0,6)$ & $1,2(0,6)$ & $1,2(0,6)$ & & \\
\hline No podía pagar el tratamiento. & $1,2(0,7)$ & $1,3(0,7)$ & $1,2(0,6)$ & & \\
\hline $\begin{array}{l}\text { Mi seguro sanitario es con una empresa privada y creía que no me } \\
\text { atenderían en la sanidad pública. }\end{array}$ & $1,1(0,5)$ & $1,1(0,5)$ & $1,2(0,5)$ & & \\
\hline No tenía tiempo disponible para ir a tratamiento. & $1,5(0,9)$ & $1,5(0,9)$ & $1,4(0,8)$ & & \\
\hline No sabía dónde pedir ayuda o asistencia. & $1,6(0,9)$ & $1,6(0,9)$ & $1,7(0,9)$ & & \\
\hline $\begin{array}{l}\text { Tenía miedo de que le diagnosticaran otra enfermedad relacionada con su consu- } \\
\text { mo de alcohol u otras drogas. }\end{array}$ & $1,9(1,0)$ & $1,9(1,0)$ & $2,1(1,1)$ & & \\
\hline $\begin{array}{l}\text { Creía que no tenía edad (era demasiado joven o demasiado mayor) para solicitar } \\
\text { tratamiento. }\end{array}$ & $1,4(0,7)$ & $1,4(0,8)$ & $1,4(0,7)$ & & \\
\hline El horario de la UCA/UA no se ajustaba a sus necesidades. & $1,4(0,8)$ & $1,4(0,8)$ & $1,5(0,9)$ & & \\
\hline EJE 4: Factores intrínsecos al tratamiento & $1,5(0,3)$ & $1,5(0,3)$ & $1,6(0,3)$ & $-2,6$ & 0,010 \\
\hline Le preocupaba que la gente pudiera verle cuando acudía a la UCA/UA. & $1,1(1,0)$ & $1,7(0,9)$ & $2,0\left(1,{ }^{\circ}\right)$ & $-2,7$ & 0,006 \\
\hline $\begin{array}{l}\text { Había tenido una mala experiencia con algún tratamiento anterior en otros tipos } \\
\text { de centros. }\end{array}$ & $1,2(0,6)$ & $1,2(0,6)$ & $1,2(0,5)$ & & \\
\hline Tenía miedo a fracasar en el tratamiento. & $2,0(1,0)$ & $2,0(1,0)$ & $2,2(1,1)$ & $-2,1$ & 0,039 \\
\hline
\end{tabular}


Pensaba que el tratamiento no se ajustaba a sus necesidades.

Le preocupaba el trato personal que fuera a recibir en la UCA/UA.

Algún conocido tuvo una mala experiencia con el tratamiento.

Le preocupaba la confidencialidad del tratamiento.

No le gusta que le pregunten sobre temas personales

Solicitó tratamiento en otros servicios (Servicios Sociales, Atención Primaria, Salud Mental, Hospital ...) pero no me enviaron a una UCA/UA.

No le gusta hablar en un grupo.

Tuvo que esperar mucho tiempo en lista de espera para recibir tratamiento.

Se sentía inseguro en una UCA/UA.

Tenía miedo de que le ingresaran en un hospital.

Solicitó tratamiento anteriormente en una UCA/UA, pero no le atendieron.

Otras personas le desaconsejaron iniciar un tratamiento.

\section{EJE 5: Estigmatización y respuesta del entorno}

Pensó que su familia no lo entendería.

Se encontraba avergonzado/a.

Pensaba que perdería a sus amigos si empezaba un tratamiento o dejaba de consumir drogas.

Consideraba que su trabajo podría peligrar si solicitaba tratamiento.

Alguien importante para usted (familiares, amigos, etc.) desaprobaban que recibiera a tratamiento.

Nadie le dijo que tuviera un problema con las drogas o le animó a que buscara ayuda.

Si acudía a tratamiento, no tenía quien cuidara de sus hijos.

Le preocupaba qué pensaría la gente de usted si solicitaba ayuda.

Iniciar un tratamiento podría generarle algún problema legal.

\begin{tabular}{|c|c|c|c|c|}
\hline $1,6(0,8)$ & $1,6(0,8)$ & $1,7(0,9)$ & & \\
\hline $1,5(0,8)$ & $1,5(0,8)$ & $1,7(0,9)$ & $-2,5$ & 0,013 \\
\hline $1,1(0,5)$ & $1,2(0,5)$ & $1,1(0,4)$ & & \\
\hline $1,7(1,0)$ & $1,7(1,0)$ & $1,9(1,1)$ & $-2,5$ & 0,014 \\
\hline $1,9(0,9)$ & $1,9(1,0)$ & $1,8(0,9)$ & & \\
\hline $1,3(0,8)$ & $1,3(0,7)$ & $1,5(1,0)$ & $-2,4$ & 0,018 \\
\hline $1,9(1,0)$ & $1,9(1,0)$ & $1,8(0,9)$ & & \\
\hline $1,2(0,5)$ & $1,2(0,5)$ & $1,2(0,5)$ & & \\
\hline $1,3(0,7)$ & $1,3(0,7)$ & $1,5(0,7)$ & $-2,3$ & 0,019 \\
\hline $1,9(1,1)$ & $1,8(1,1)$ & $2,0(1,1)$ & & \\
\hline $1,0(0,3)$ & $1,0(0,3)$ & $1,1(0,3)$ & & \\
\hline $1,2(0,6)$ & $1,2(0,6)$ & $1,2(0,7)$ & & \\
\hline $1,6(0,4)$ & $1,5(0,4)$ & $1,6(0,4)$ & $-2,1$ & 0,035 \\
\hline $1,8(1,0)$ & $1,7(0,9)$ & $1,9(1,0)$ & & \\
\hline $2,2(1,1)$ & $2,1(1,1)$ & $2,6(1,2)$ & $-4,8$ & 0,000 \\
\hline $1,5(0,8)$ & $1,5(0,8)$ & $1,4(0,8)$ & & \\
\hline $1,7(1,0)$ & $1,7(1,0)$ & $1,6(1,0)$ & & \\
\hline $1,1(0,5)$ & $1,1(0,5)$ & $1,2(0,5)$ & & \\
\hline $1,8(1,0)$ & $1,9(1,1)$ & $1,6(1,0)$ & 2,9 & 0,004 \\
\hline $1,2(0,6)$ & $1,1(0,5)$ & $1,3(0,7)$ & $-2,1$ & 0,034 \\
\hline $1,7(0,9)$ & $1,6(0,9)$ & $2,0(1,1)$ & $-3,7$ & 0,000 \\
\hline $1,1(0,5)$ & $1,1(0,4)$ & $1,2(0,5)$ & & \\
\hline
\end{tabular}

\section{DISCUSIÓN}

Los resultados del presente estudio muestran que la demanda en los Centros de Atención Primaria es el recurso más utilizado previamente a la asistencia a centros específicos en drogodependencias, de manera que el $36,1 \%$ de los entrevistados solicitó tratamiento con anterioridad en este tipo de recursos. Estos datos ya habían sido reflejados en estudios realizados en otras comunidades autónomas y a nivel internacional ${ }^{36,37}$. La Atención Primaria proporciona un entorno excelente para el diagnóstico e intervención precoz ${ }^{38}$ y puede minimizar la posible barrera de accesibilidad que supone el desconocimiento de los recursos de tratamiento existentes y su funcionamiento ${ }^{25}$. Probablemente por esta razón, el eje "factores extrínsecos al tratamiento", que engloba los ítems relacionados con el desconocimiento de los recursos y su funcionamiento, es el que obtiene una menor puntuación. 
Aproximadamente un $18 \%$ de la muestra había acudido previamente a servicios de Salud Mental, siendo las mujeres las que más habían utilizado estos recursos. Entre los motivos que pueden justificar esta situación están que las mujeres cuando desarrollan problemas relacionados con el abuso de alcohol, lo hacen de una manera más rápida y grave que los hombres ${ }^{12}$, lo que podría verse reflejado en un aumento del riesgo de comorbilidad psiquiátrica ${ }^{14}$. Además, tienden a abusar del alcohol cuando están solas, bajo presión de su pareja alcohólica y bajo condiciones de estados emocionales negativos, mientras que los hombres son más propensos a beber en exceso en situaciones sociales y bajo condiciones de estados emocionales positivos ${ }^{37}$. Este comportamiento en las mujeres responde a una conducta de huida o evitación y propicia el proceso de autoculpabilización y vergüenza cuando el consumo abusivo genera una dependencia y se convierte, de esta manera, en un obstáculo para la demanda de tratamiento.

Los pacientes que no habían acudido previamente a un servicio sanitario por su consumo de drogas puntuaban más bajo en el eje "no conciencia de enfermedad y problemas asociados". De hecho, este perfil de pacientes no solo no niega sus preocupaciones sobre su hábito, sino que además reconocen que beben demasiado e intentan, o al menos consideran, reducir su consumo ${ }^{39}$. Probablemente estos sujetos son conscientes de su adicción, y por tanto, acuden directamente a recursos específicos como las UCA y las UA. Asimismo, la asunción del consumo abusivo como adicción y, por ende de enfermedad, propició que puntuaran más bajo en los ejes: a) resistencia al cambio, ya que estaban suficientemente motivados para iniciar un tratamiento; b) factores intrínsecos al tratamiento, pues asumían los elementos tanto positivos como negativos del mismo; y c) estigmatización, ya que se consideraban enfermos y, por tanto, no tenían reparo en admitir que estaban en tratamiento

Las principales barreras de accesibilidad al tratamiento en la población con dependencia de alcohol estudiada son "la no conciencia de enfermedad ni de problemas asociados" y "la resistencia al cambio", con puntuaciones medias de 2,2 y 2,0, respectivamente, siendo los hombres los que más puntuaban en el eje "no conciencia de enfermedad". Estos pacientes podrían por tanto beneficiarse de intervenciones educativas que incrementaran su conocimiento sobre los síntomas de abuso o dependencia del alcohol, sus consecuencias y la disponibilidad y efectividad de los servicios de tratamiento ${ }^{40}$.

Se obtuvieron bajas puntuaciones en factores intrínsecos y extrínsecos relacionados con el tratamiento tales como la desaprobación por parte de algún familiar o amigo a comenzar un tratamiento. Esto contrasta con estudios previos que destacan el importante papel que juegan las redes sociales de los adictos al alcohol como elemento disuasorio del acceso al tratamiento, especialmente debido al consumo de sustancias por parte de la pareja ${ }^{41-43}$.

La "estigmatización y respuesta del entorno" y los "factores intrínsecos al tratamiento" obtuvieron puntuaciones más altas en mujeres que en hombres. Las barreras relacionadas con la estigmatización y que obtuvieron una mayor puntuación fueron sentirse avergonzado, la preocupación ante lo que pensara la gente por la solicitud de ayuda o que pudieran verle cuando acudía a la UCA/UA; si bien esta barrera era considerada en el eje "factores intrínsecos al tratamiento" también podría estar englobada en el eje "estigmatización y respuestas al tratamiento". El proceso de estigmatización que sufren las mujeres dependientes del alcohol ha sido definido por otros autores ${ }^{44}$ y puede ser una de las causas de que los hombres estén más predispuestos a buscar tratamiento y de que las mujeres dependientes del alcohol que no utilizan los servicios de tratamiento no manifiesten necesidad de ayuda ${ }^{40}$. Todo ello se ve reflejado en una escasa representación de la mujer en los centros de tratamiento específicos ${ }^{25}$. Otro de los motivos que origina que las mujeres acudan en menor número a centros de tratamiento es la escasa derivación de las mujeres con dependencia de alcohol a recursos especializados desde el ámbito laboral y escolar ${ }^{45}$. Respecto a los factores intrínsecos, estudios previos muestran que las mujeres señalan como posibles barreras el desconocimiento de los recursos existentes para el tratamiento de la dependencia de alcohol y los problemas que supone el acceso al tratamiento para su vida cotidiana, fundamentalmente cuando tienen hijos a su cargo ${ }^{17,46}$.

El presente estudio tiene varias limitaciones. La muestra procedía de población que ya se encontraba en algún programa de tratamiento ambulatorio en centros especializados en drogodependencias y que, por tanto, ya habían podido superar las barreras, lo que podría implicar que se infraestimase la importancia de determinados factores. No obstante, no se observaron diferencias en las barreras de tratamiento según el tiempo que llevaban los pacientes en tratamiento en la unidad. Otra limitación podría ser la complacencia del paciente respecto al profesional y el sistema sanitario; por ello, se intentó no inducir ni influir en las respuestas de los sujetos. Además, tampoco se ha considerado la comorbilidad psiquiátrica asociada, la cual puede ser una barrera para acceder al tratamiento en hombres y, sobre todo, en mujeres, pues presentan una mayor prevalencia y severidad de trastornos de ansiedad y depresión ${ }^{32}$. Por otra parte, toda la información del presente estudio se recogió mediante un cuestionario estructurado, no teniendo en consideración datos cualitativos que pudieran proporcionar una mayor información sobre las respuestas 
de los entrevistados y aportar factores no considerados en el estudio.

El presente trabajo muestra por tanto que existen diferencias intergénero en las barreras que retrasan el acceso a tratamiento. Sería conveniente elaborar instrumentos y protocolos de intervención adaptados y optimizados según el género, que se utilizaran en los centros en los que habitualmente buscan ayuda las personas con problemas relacionados con el consumo de alcohol, es decir, centros de Atención Primaria y de Salud Mental, para facilitar su detección y derivación a centros específicos de tratamiento. Además, futuros estudios deberían incluir investigación cualitativa y recoger información sobre las barreras al tratamiento de aquellas personas que todavía no han accedido a centros especializados.

\section{AGRADECIMIENTOS}

Los datos del presente estudio se obtuvieron como parte de una investigación financiada por la Fundación para el Estudio, Prevención y Asistencia a las Drogodependencias (FEPAD). Asimismo hay que agradecer a los profesionales de las Unidades de Conductas Adictivas y Unidades de Alcohología su colaboración activa en el pase de las encuestas.

\section{REFERENCIAS}

1. López-Marina V, Pizarro G, Alcolea R, Beato P, Galindo E, Montellà N. Evaluación del cribado y la efectividad de una intervención breve en bebedores de riesgo atendidos en consultas de atención primaria. Aten Primaria 2005; 36: 261-8.

2. Organización Mundial de la Salud. The World Health Report 2002. Reducing risks, promoting healthy life. Ginebra: World Health Organization; 2003.

3. Navarro J, Megías E (dirs). El consumo de drogas y factores asociados en la Comunidad Valenciana (II). Valencia: Generalitat Valenciana; 1999.

4. Fundación de Ayuda contra la Drogadicción. Informe técnico: avance de resultados generales del consumo de drogas en la Comunidad Valenciana 2004. Madrid: FAD; 2005.

5. Ley 3/1997 de 6 de junio, sobre drogodependencias y otras conductas adictivas de la Comunidad Valenciana. Valencia: Generalitat Valenciana; 1997.

6. Tomás S. Balance de situación del abordaje de los trastornos adictivos en la Comunidad Valenciana. Ponencia presentada en el Congreso Nacional "Sociedad de consumo ¿de drogas?" de la Universidad Cardenal Herrera-CEU. Valencia, 3 - 5 de noviembre de 2005.
7. Mosquera J, Guimerans C, Gómez B, Davila N. Desintoxicación ambulatoria de alcohol: una opción segura. Adicciones 2005; 17: 29-32.

8. Gual A, Lligoña A, Colom J. Five-year outcome in alcohol dependence. A Naturalistic study of 850 patients in Catalonia. Alcohol Alcoholism 1999; 34: 183-92.

9. Holder $\mathrm{H}$, Blose JO. The reduction of health care costs associated with alcoholism treatment: A 14-year longitudinal study. J Stud Alcohol 1992; 53: 293-302.

10. Bunn JY, Booth BM, Loveland CA, Blow FC, Fortney JC. The relationship between mortality and intensity of inpatient alcoholism treatment. Am J Pub Health 1994; 84: 211-214.

11. Arias F, Sánchez S. Disminución del uso de recursos sanitarios tras un programa de tratamiento del alcoholismo. Adicciones 2003; 15: 17-21.

12. Green CA. Gender and use of substance abuse treatment services. Alc Res Health 2006; 29: 55-62.

13. Moos RH, Moos, BS, Timko C. Gender, treatment and self-help in remission from alcohol use disorders. Clin Med \& Res 2006; 4: 163-74.

14. Weiss SRB, Kung HC, Pearson JL. Emerging issues in gender and ethnic differences in substance abuse and treatment. Current Woman Health Reports 2003; 3: 245-53.

15. Kane RL, Wall M, Potthoff S, Stromberg K, Dai Y, Meyer ZJ. The effect of alcoholism treatment on medical care use. Med Care 2004; 42: 395-402.

16. Kessler RC, Zhao S, Katz SJ, Kouzis AC, Frank RG, Edlund M, Leaf P: Past-year use of outpatient services for psychiatric problems in the National Comorbidity Survey. Am J Psychiatry 1999; 156: 115-23.

17. Grant BF. Barriers to alcoholism treatment: reasons for not seeking treatment in a general population sample. J Stud Alcohol 1997; 58: 365-71.

18. Booth BM, Cook CA, Blow FC, Bunn JY. Utilization of outpatient mental health services after inpatient alcoholism treatment. J Ment Health Adm 1992; 19: 21-30

19. Aldama E, Ariño J, Ballesteros J, Segovia M, Gutiérrez M. Utilización de servicios psiquiátricos de alcoholismo en un seguimiento a 18 meses de varones alcohólicos. Med. Clin 1996; 106: 768-75.

20. Olfson M, Kessler RC, Berlung PA, Lin E. Psychiatric disorder onset and first treatment contact in the United States and Ontario. Am J Psych 1998; 155: 1415-22.

21. Wang PS, Berglund PA, Olfson M, Kessler RC. Delays in initial treatment contact after first onset of a mental disorder. Health Serv Res 2004; 39: 393-415.

22. Donabedian A. Evaluating the quality of medical care. Milbank Memorial Fund Quarterly 1966; 44; 166-202.

23. Hall JA, Dornan MC. What patient like about their medical care and how often they are asked: a meta-analysis of the satisfaction literature. Soc Sci Med 1988; 27: 935-9.

24. Martin JC. Opinión de los usuarios sobre la atención prestada en un Centro Ambulatorio de Atención a Drogodependientes. Adicciones 2003; 15: 341-350. 
25. Saunders SM, Zygowicz KM, D'Angelo BR. Personrelated and treatment-related barriers to alcohol treatment. J Subst Abuse Treat 2006; 30: 261-70.

26. Weisner C, Matzger H, Kaskutas LA. How important is treatment? One-year outcomes of treated and untreated alcohol-dependent individuals. Addiction 2003; 98: 901-11.

27. López-Torrecillas F, Verdejo A, Muñoz-Rivas MJ, Caballo V, Graña JL. Variables de personalidad que motivan hacia el tratamiento. Adicciones 2005; 17: 315-23.

28. Marion TR, Stefanik-Campisi C. The elderly alcoholic: identification of factors that influence the giving and receiving of help. Perspect Psychiatr Care 1990; 25: 32-5.

29. Wenzel SL, Koegel P, Morton SC, Miu A, Jinnett KJ, Greer Sullivan J. Access to inpatient or residential substance abuse treatment among homeless adults with alcohol or other drug use disorders. Med Care 2001; 39: 1158-69.

30. Fortney JC, Blow FC, Bunn JY. . The effects of travel barriers and age on the utilization of alcoholism treatment aftercare. Am J Drug Alcohol Abuse 1995; 21: 391-406.

31. Desai MM, Rosenheck RA, Craig TJ. Screening for alcohol use disorders among medical outpatients: the influence of individual and facility characteristics. Am J Psychiatry 2005; 162: 1521-6.

32. Brady TM, Ashley OS (eds). Women in substance abuse treatment: results from the Alcohol and Drug Services Study (ADSS). Rockville: Substance Abuse and Mental Health Service Administration; 2005.

33. Thom B. Sex differences in help-seeking for alcohol problems. The barriers to help-seeking. Br J Addict 1986; 81: 777-88

34. Substance Abuse and Mental Health Service Administration. 2002 National Survey on Drug Use and Health. Washington D.C.: SAMHSA; 2003.

35. Miller RW, Tonigan JS. The Barriers Questionnaire. Center on Alcoholism, Substance Abuse, and Addictions. Alburquerque: University of New Mexico; 2002.
36. Gómez-Talegón MT, Álvarez FJ. Demand for prior treatment in alcoholic patients. Subst Use \& Misuse 2006; 41: 61-9.

37. Schober R, Annis HM. Barriers to help-seeking for change in drinking: a gender-focused review of the literature. Addict Behav 1996; 21: 81-92.

38. Coulton, S, Drummond, C, James D, Godfrey, C, Bland, M, Parrott, S, Peters, T, Stepwice Research Team. Opportunistic screening for alcohol use disorders in primary care: comparative study. BMJ 2006; 332: 511-7.

39. Williams EC, Kivlahan DR, Saitz R, Merrill JO, Achtmeyer CE, McCormick KA, Bradley KA. Readiness to Change in Primary Care Patients Who Screened Positive for Alcohol Misuse. Ann Fam Med. 2006; 4: 213-20.

40. Wu LT, Ringwalt CL. Alcohol dependence and use of treatment services among women in the community. Am J Psychiatry 2004; 161: 1790-7.

41. Bendtsen P, Dahlstrom ML, Bjurulf P. Sociodemographic gender differences in patients attending a communitybased alcohol treatment centre. Addict Behav 2002; 27: 21-33.

42. Grella CE, Joshi V. Gender differences in drug treatment carrers among clients in the National Drug Abuse Treatment Outcome Study. Am J Drug Alcohol Abuse 1999; 25: 385-406.

43. Lex BW. Gender differences and substance abuse. Adv Subst Abuse 1991; 4: 225-96.

44. Wilsnack SC, Murray MM. Alcohol Abuse and Stigma. Comunicación presentada en "Stigma and Global Health: an International Conference". Bethesda, 5-7 septiembre, 2001.

45. Morgentern J, Bux DA. Examining the effects of sex and ethnicity on substance abuse treatment and mediational pathways. Alcohol Clin Exp Res 2003; 27:1330-2.

46. Gómez J. El alcoholismo femenino: una perspectiva sociológica. Valencia; Rialla editores: 2005. 